K urz vor Fertigstellung dieses Heftes verstarb bei einem tragischen Unfall Herr Prof. Dr. Georg Becker, Direktor der Neurologischen Universitätsklinik Homburg/Saar. Die deutsche Neurologie hat einen herausragenden Vertreter des Faches, visionären Wissenschaftler und wunderbaren Menschen verloren. Mit großem Respekt widmen wir dieses Heft Herrn Prof. Becker in tiefer Trauer.

\title{
Die Parkinson-Therapie ist spannend
}

$\mathrm{D}$ as Parkinson-Syndrom ist eine der häufigsten neurologischen Krankheiten und wird in der Zukunft weiter an Bedeutung gewinnen. Die diagnostischen und therapeutischen Möglichkeiten haben in den letzten zehn Jahren erstaunlich zugenommen, so dass heute die Diagnostik sicherer und die Therapie effizienter ist als bei den meisten anderen neurologischen Erkrankungen. Selbst wenn man sich schwerpunktmäßig mit der Erkrankung beschäftigt, ist es fast unmöglich alle Neuerungen sofort in der tägliche Praxis umzusetzen. Deshalb muss das Wissen ständig und in seiner gesamten Breite aktualisiert werden. Durch das große Angebot von Fortbildungsveranstaltungen wird suggeriert, dass eine umfassende Information erfolgt. Dies ist jedoch nicht möglich, weder in einer Abendveranstaltung, noch in einem Wochenendseminar. Dies wird uns auch nicht mit unserem Schwerpunktheft gelingen.

Viel mehr versuchen wir, die wichtigsten Aspekte der Diagnostik und medikamentösen Therapie in Übersichtsarbeiten darzustellen und auch neue Gesichtspunkte wie die Zelltransplantation und tiefe Hirnstimulation und die klinisch relevante, aber weniger oft dargestellte medikamentösen Interaktionen pragmatisch zu vermitteln. Selbstverständlich handelt es sich bei dem Heft nur um eine Momentaufnahme, die bereits in wenigen Monaten in Einzelaspekten aktualisiert werden muss. Deshalb sehen Sie unsere Beiträge als einen Schritt in Ihrer kontinuierlichen medizinischen Fortbildung (CME) an und kontrollieren Sie sich selbst, in dem Sie die gestellten Fragen beantworten.

Wir wünschen Ihnen viel Spaß beim Lesen und hoffen Ihre fachlichen Erwartungen zu erfüllen.

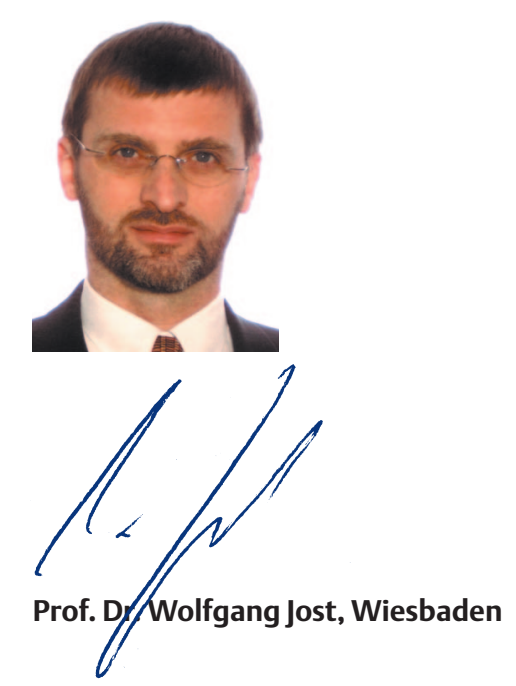

